\title{
Ectopia Cordis: Two Different Cases with Literature Review
}

\author{
Manoj Kumar Sahu ${ }^{1}$ Mayank Yadav ${ }^{1}$ Yatin Arora ${ }^{1}$ Sarveshpal Singh ${ }^{1}$ Velayoudam Devagourou ${ }^{1}$ \\ Shiv Kumar Chaudhary ${ }^{1}$
}

${ }^{1}$ Intensive Care for CTVS, Department of CTVS, CN Centre, All India Institute of Medical Sciences, New Delhi, India

\begin{abstract}
Address for correspondence Manoj Kumar Sahu, MD, DNB, Intensive Care for CTVS, Department of CTVS, CN Centre, All India Institute of Medical Sciences, New Delhi 10029, India

(e-mail: drmanojsahu@gmail.com).
\end{abstract}
Abstract
Keywords
- ectopia cordis
- surgical correction
- literature review

Ectopia cordis is a rare congenital anomaly with thoracic exteriorization of the heart. With few exceptions, it is universally fatal. Surgical correction is the only final treatment option for this defect but with a very high mortality. The authors present two cases of ectopia cordis, both were operated upon in urgent basis, and one baby survived.

\section{Introduction}

Ectopia cordis (EC) is a rare congenital cardiac malformation defined as a defect in the anterior chest wall and/or abdominal wall with abnormal placement of the heart outside the thoracic cavity with associated defect in the parietal pericardium, diaphragm, sternum, and heart. ${ }^{1}$

The incidence of EC is 5.5 to 7.9 per 1 million live births and includes $0.1 \%$ of congenital heart diseases. ${ }^{2-4}$ Despite profound advances in surgery, anesthesia, and intensive care, complete thoracic/thoracoabdominal EC poses a tough surgical challenge with only few long-term survivors. ${ }^{5-7}$ Here, we report two cases, one with complete thoracoabdominal type (pentalogy of Cantrell) and the other with partial thoracic EC. Both had intracardiac defects.

\section{Case 1}

A 4-day-old female neonate, weighing $2.9 \mathrm{~kg}$, was admitted to the intensive care unit (ICU) of a tertiary care hospital, with protrusion of heart through the anterior chest wall defect at birth. There was no history of breathing difficulty, cyanosis, any other body part defect, and no family history of congenital defect. The mother, a nonsmoker and nonalcoholic, did not undergo a proper antenatal checkup, and she had no history of ingestion of any unprescribed medications. This was the second baby born to a nonconsanguineous marriage, and it was a normal vaginal home delivery without any complications.
On examination, the baby looked ill, had fever $\left(100^{\circ} \mathrm{F}\right)$, was anicteric, and tachypneic. There was a clear visible midline anterior chest wall defect measuring $6 \times 4 \mathrm{~cm}$ in the craniocaudal and horizontal diameters extending between the nipples with an ulcer extending from the defect to the umbilical stump. The whole heart with irregular contour was outside the thoracic cavity leaving only the major vessels inside the chest wall (Video 1).

\section{Video 1 \\ Case 1 (intubated and on controlled ventilation) with complete ectopia cordis with omphalocele (pentalogy of Cantrell) preoperatively. Online content is viewable at: https://www.thiemeconnect.com/products/ejournals/ html/10.1055/s-0039-1684882.}

The neonate had respiratory rate (RR) 88 breaths/min, heart rate (HR) 162 beats/min, blood pressure (BP) 68/34 mm Hg, and oxygen saturation $\left(\mathrm{Spo}_{2}\right)$ 94\%. She was awake, irritable, crying, with normal primitive reflexes and no other midline defects. A diagnosis of complete thoracoabdominal EC (pentalogy of Cantrell), with presumed sepsis (fever, respiratory distress, ulcer on the defect) was made. Laboratory reports showed neutrophilic predominant (82\%) higher total leucocyte count $19,000 / \mathrm{mm}^{3}$, platelets $=198,000 / \mathrm{mm}^{3}$. Her prothrombin time-to-international normalization ratio and serum biochemistry were normal. Epicardial echocardiogram detected a

Copyright @2018 Official Publication of The Simulation Society (TSS), accredited by International Society of Cardiovascular Ultrasound (ISCU).
License terms

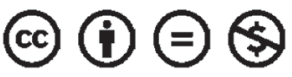


secundum atrial septal defect (ASD) and one small ventricular septal defect (VSD) with normal cardiac function.

A multistaged surgery was planned for this baby-an attempt to accommodate the heart in the thoracic cavity failing which to cover the heart and repair the abdominal defect was decided in this first stage. She was already on broad-spectrum antibiotic coverage with cefotaxime and amikacin, which were changed over to piperacillin + tazobactam (50 $\mathrm{mg} / \mathrm{kg} / 6 \mathrm{~h}$ ) and teicoplanin (10 mg/kg/once daily).

Intraoperatively, trial approximation of pericardium caused severe hemodynamic compromise, and it was not possible, so the heart was covered with PTFE (polytetrafluroethylene) graft. The abdominal defect along with the protruded bowel loops was covered with bovine pericardium. A sterile blood bag was oversewn above both the PTFE graft and bovine pericardium as a guard against desiccation and infection ( - Fig. 1 ). The baby was shifted to the ICU on very high doses of inotropes (adrenaline and nor-adrenaline $0.02 \mu \mathrm{g} / \mathrm{kg} / \mathrm{min}$ each,

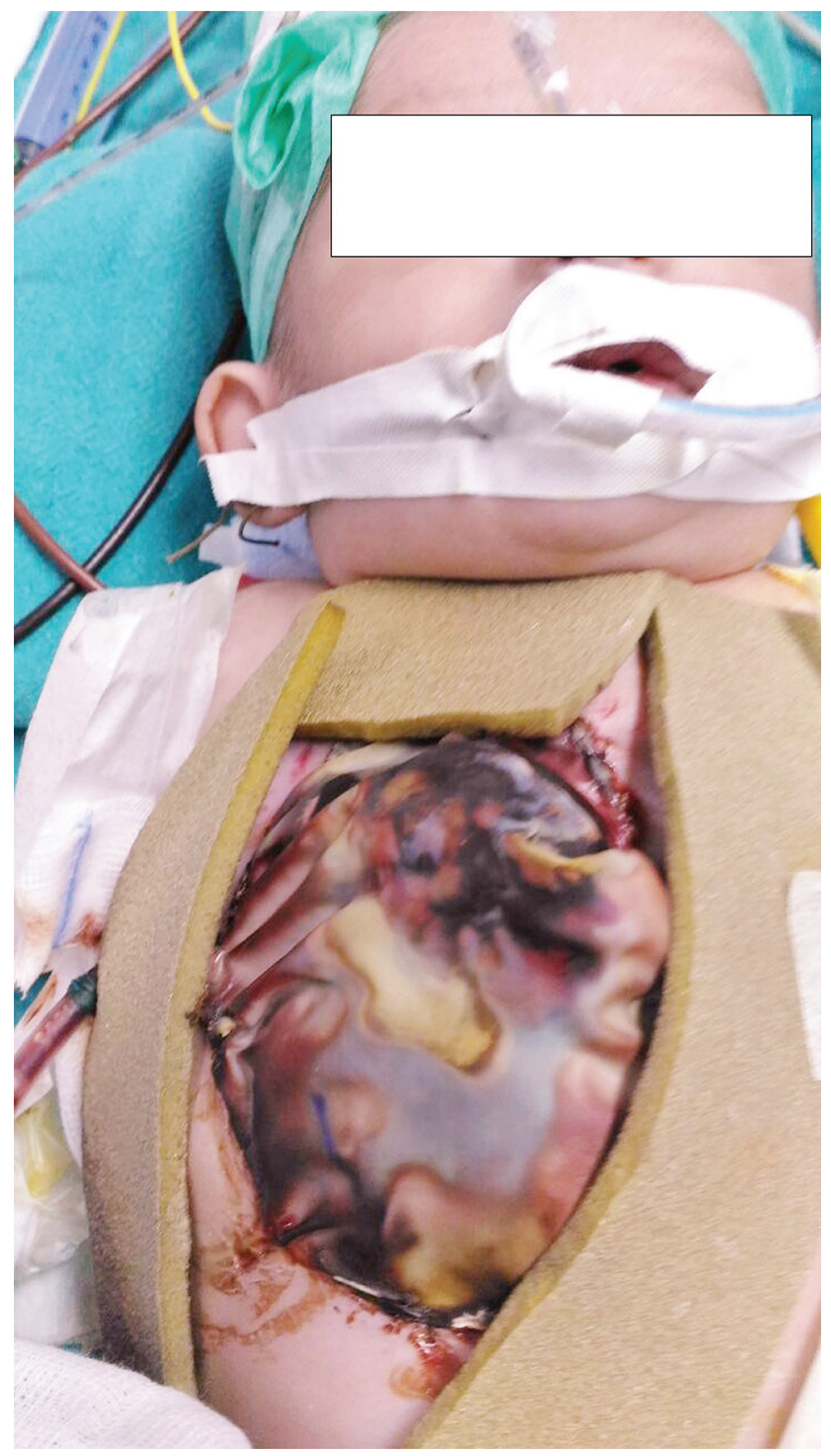

Fig. 1 Pentalogy of Contrell: Primary coverings using PTFE (polytetrafluoroethylene) graft and bovine pericardium for chest and abdominal defects respectively. Both the primary covers are again covered by sterile blood bag. dopamine and dobutamine $10 \mu \mathrm{g} / \mathrm{kg} /$ min each) with unstable hemodynamics (BP 45/30 mm Hg, HR 176 beats/min, high central venous pressure of $17 \mathrm{~cm} \mathrm{H} \mathrm{O}$, severe metabolic acidosis $\mathrm{pH} 7.12, \mathrm{HCO}_{3} 11$, base deficit -15 , lactate of 8.7 , and low urine output). Her hemodynamics continued to remain critically balanced with inotropic adjustments and correction of metabolic acidosis, but she became anuric and acidosis increased within next 2 hours. Peritoneal dialysis (PD) was initiated with $10 \mathrm{~mL} / \mathrm{kg}$ of dialysate at 1 hourly cycles. The child remained steady at a systolic BP of 40 to $45 \mathrm{~mm} \mathrm{Hg}$ for few hours, with improvement in acidosis by sodium bicarbonate therapy and PD, but she rapidly deteriorated after 8 hours of surgery, had cardiac arrest, and could not be resuscitated.

\section{Case 2}

A full-term, 3-day-old male neonate was delivered vaginally at 40 weeks of gestation in a primary health care setup with birth weight of $3 \mathrm{~kg}$. The baby was referred to our ICU with visible cardiac pulsation in upper part of the thorax with overlying intact skin. He was the third child of the 24-year-old mother, born to a nonconsanguineous couple. Drug exposure during this pregnancy was ruled out, and antenatal course was uneventful. There were only two prenatal consultations, and no fetal ultrasound was done till delivery.

On examination, the newborn was found to be alert and responsive, tachypneic (RR 66 breaths/min). Paradoxical indrawing of chest wall during inspiration was present. His HR was 155 beats/min, BP was 68/35 mm Hg, and systolic murmur was found on auscultation of the precordium. There was a big gap in the anterior chest wall, bony defect on the upper two-thirds of sternum with intact overlying skin. The heart was seen protruding through the defect during respiration (Video 2). No other visible defects were found on

\section{Video 2}

Case 2-partial ectopia cordis with intact skin preoperatively. Online content is viewable at: https:// www.thiemeconnect.com/products/ejournals/ html/10.1055/s-0039-1684882.

the abdomen, neck, or extremeties. Echocardiography diagnosed a small secundum ASD and normal cardiac function. The abdominopelvic and transfontanellar ultrasounds were normal. Routine blood profile and serum chemistry were unremarkable.

A diagnosis of partial thoracic EC was made. The newborn was monitored in the ICU and was taken up for first-stage repair of the defect next morning by the team of cardiac and plastic surgeons under general anesthesia.

Through a midline thoracic incision, pericardium was approximated toward each other and the heart was covered. Then a myocutaneous flap was dissected on both side of midline, approximated, and sutured in the midline, and lastly, the skin was sutured ( - Fig. 2). The neonate was shifted to ICU with stable hemodynamics, without any inotropic support. 


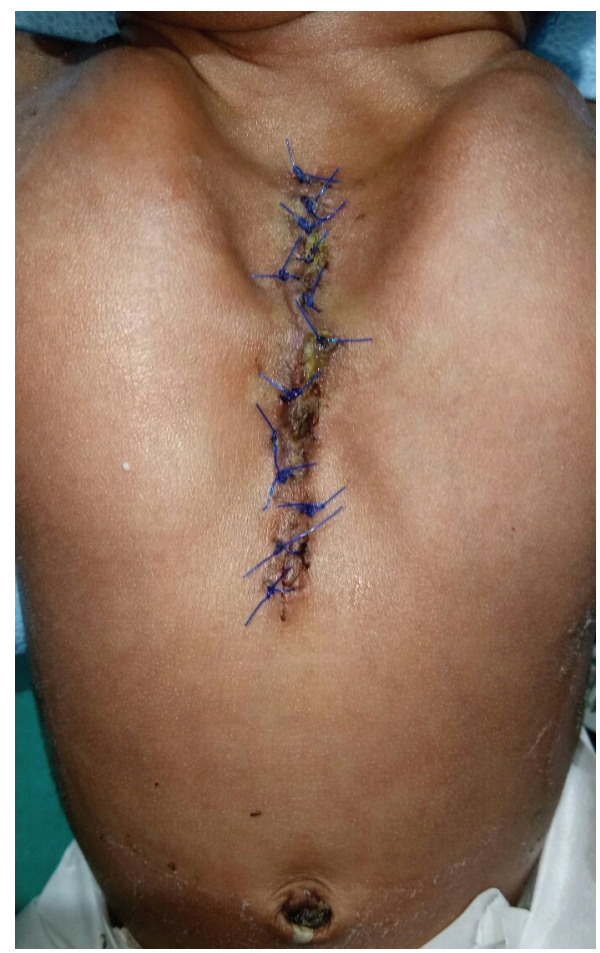

Fig. 2 Case 2 after first-stage repair of the defect (partial ectopia cordis) postoperatively.

His postoperative course remained relatively unremarkable. The baby was weaned from ventilatory support and extubated after 18 hours. He remained tachypneic after extubation (RR 55 breaths/min), but arterial blood gases, $\mathrm{Spo}_{2}$, and hemodynamics were stable. He was discharged from ICU on third postoperative day and from the hospital on day 12. Karyotyping was not done in both the cases due to financial constraints.

\section{Discussion}

Ectopia cordis always remains tough for surgical correction, challenging for intensive care management, and mostly associated with poor outcomes. In a complete EC, the heart is entirely outside the thoracic cavity with or without a pericardial covering, may also be associated with abdominal defects such as in Cantrell's defect, and constitutes a neonatal emergency. In a partial EC, the heart can be seen to pulsate through the skin with no external skin defect, so it can be considered for a planned surgery but at urgent basis.

The first known case of EC was reported by Stensen in $1671 .^{8-10}$ It was first described by Haller in 1706 and classified into five types by Weese in 1818 and Todd in $1836^{8}$ : cervical (3\%), cervicothoracic $(<1 \%)$, thoracic $(60 \%)$, thoracoabdominal (7\%), and abdominal (30\%). Thoracic defects are most commonly reported type. ${ }^{8}$

Pentalogy of Cantrell-the syndromic form of EC-consists of five associated anomalies: distal sternal defect, midline supraumbilical abdominal wall defect, ventral diaphragmatic hernia, defect in the apical pericardium, free communication into the peritoneal cavity, and congenital intracardiac defect. ${ }^{10}$ It is extremely rare and usually incompatible with life. Although EC is considered as an isolated, sporadic malformation, some abnormal karyotypes such as trisomy 18 , Turner's syndrome, $46 \mathrm{XX}$, and $17 \mathrm{q}$ + have been reported. ${ }^{9}$

Ectopia cordis is frequently associated with other congenital defects involving multiple organ systems, including intracardiac defects (80.2\%), that is, VSD $100 \%$, ASD $53 \%$, tetralogy of Fallot (TOF, 20\%), left ventricular diverticulum (LVD, 20\%), and pulmonary hypoplasia. ${ }^{6}$ Newborn with these complex and life-threatening anomalies require intensive care management right from birth due to the risk of heart failure, hypoxia, and infection. To prevent desiccation of exposed heart and viscera, heat loss, dehydration, and infection, it is recommended to cover them with-saline soaked gauze pads, and these neonates should be resuscitated immediately to prevent rapid deterioration in their condition. ${ }^{11}$

The final management of the condition is urgent surgical correction. The first attempted repair of EC was performed in 1925 by Cutler and Wilens. ${ }^{12}$ Koop (1975) achieved the first successful repair of thoracic EC in two stages..$^{13}$ Amato et al reported a successful single-stage repair of thoracic EC in $1995 .{ }^{14}$ The thoracic cavity is small in most of these cases with little mediastinal space for the heart. Attempt to close the chest wall or even pericardial cover of the heart often results in hemodynamic embarrassment secondary to kinking of the great vessels or compression of the heart. This is what happened in our first case. Trial attempt to cover the heart with pericardium caused severe hemodynamic compromise, so failed. There was neither possibility of reducing the heart into the thoracic cavity nor any chance of attempting to repair the intracardiac defects at the same sitting.

Aim of surgical treatment is to (1) provide soft tissue cover of the heart, (2) reduce the heart into the thoracic cavity, (3) provide palliation and repair of any intracardiac defect, and (4) perform reconstruction of chest wall.

During surgical repair of EC, if primary approximation with skin flaps cannot be achieved or if severe hemodynamic instability occurs, a split-thickness skin graft, or cadaveric skin graft, or prosthetic materials can be sutured to the skin edges. The defect can then be reduced slowly over the subsequent weeks. The reduction in the heart into the thoracic cavity is performed at subsequent stages. Simple stable intracardiac defects such as ASD, VSD, LVD, and TOF can be repaired at the same time, provided the defect is not infected unlike our first case that was possibly infected. Chest wall reconstruction is performed at a later date.

The role of antenatal ultrasound in EC is not only important for parental counseling, planning delivery, and postnatal management, but it may also provide parents with an option of termination of pregnancy if diagnosis is made before fetal viability, in view of its extremely poor prognosis especially the complete type of EC. ${ }^{8}$

Prognosis: The various clinical types of EC have different prognosis. Cervical and thoracic ECs are usually fatal within days, because the heart is exposed and malformed. Abdominal EC carries a better prognosis, probably because intracardiac abnormalities are rarer and the absence of omphalocele reduces the morbidity and mortality. ${ }^{15}$ Cantrell's defect is extremely rare and usually incompatible with life; 
thus, its exact incidence is not found in the literature. In this condition, the heart is uncovered in $41 \%$, covered with serous membrane in $31 \%$, and with skin in $27 \%$, as described in a particular case series. ${ }^{16-18}$ In our first case, a thoracoabdominal EC, the heart was uncovered and the defect extended down to the umbilical region. Survival remains significantly worse in the presence of severe EC (5-10\%). ${ }^{19,20}$ In a retrospective case series with seven patients, O'Gorman and colleagues reported survival in four cases at a mean of 60.5 months of follow-up. Care was withdrawn in the remaining three patients due to lack of improvement in cardiorespiratory status or an inoperable cardiac lesion. ${ }^{21}$ Surviving patients have significant morbidity due to the multisystem compromise.

In a report spanning a period of more than a decade, of 13 patients with EC, 5 survived beyond infancy. None of the survivors had significant extrathoracic defects. All patients who did not survive had associated omphalocele, pulmonary hypoplasia, or pentalogy of Cantrell. ${ }^{22}$ Cervical EC appears to be incompatible with life. ${ }^{23}$

Our second case survived because the baby had a safer partial defect with intact skin, without infection, and he reached us at the earliest possible time and was operated upon urgently, but the first child with complete EC (Cantrell's defect) expired 8 hours after surgical closure with PTFE graft of the externalized heart due to graft desiccation and sepsis. Extracorporeal membrane oxygenation (ECMO $)^{24}$ could have been tried in this case to have a better probable outcome, but for fear of sepsis and cost, it was not possible to implement. Children with pentalogy of Cantrell are sickest before surgery. They may have pulmonary hypoplasia, unstable metabolically, and hypoxic, and require high inotropic support. Venoarterial (VA) ECMO, if established before surgery, will stabilize these neonates metabolically, hemodynamically, and also respiratory failure will be avoided. Usually VA ECMO with central cannulation strategy is most ideal for these smaller babies. And once on VA ECMO, the surgical repair would probably also become easier as the hemodynamic stability could be maintained while accommodating the heart within the thoracic cavity. Postoperative requirement of inotropes will be less, heart failure and renal failure due to hypoperfusion would be avoided, and the neonates would be weaned off ECMO gradually. Thus VA ECMO is a useful modality in managing these children in the perioperative period when they become very unstable in spite of other optimal medical therapy and mechanical ventilation. Institution of ECMO in these small babies is mostly done by central cannulation of the right atrium for venous drainage and aorta or carotid artery for return of oxygenated blood. ${ }^{24}$ Also, neonates with congenital diaphragmatic hernia with hypoplastic lungs require venovenous (VV) ECMO support in the perioperative period when the conventional medical therapy becomes insufficient to stabilize them. ${ }^{24}$ Newborns with meconium aspiration syndrome also can be salvaged with VV ECMO failing maximal ventilation and medical therapy. Though there are no adequate references about the use of ECMO in the surgical repair of EC, it can be used as a bridge for surgical repair.

Complications after surgical repair of complete EC are mainly hemodynamic issues in the early postoperative period, for example heart failure and low cardiac output
(LCO) syndrome, requirement of high inotropic support, other organ dysfunction such as acute renal failure and liver dysfunction due to hypoperfusion secondary to LCO, requirement for perintoneal dialysis, desiccation of the artificial graft (PTFE) covering the heart (sometimes the PTFE graft gets adhered to the heart behaving like constrictive pericarditis), requirement of prolonged mechanical ventilation leading to respiratory tract infections (multidrug-resistance gram-negative ventilator-associated pneumonia), wound infection, and sepsis. Late complications are few and may be limited to bony thoracic cage deformity. Children with partial ectopia such as our second case may recover uneventfully in the postoperative period. They usually do not require inotropic support, but weaning from mechanical ventilation should be done very slowly and gradually. They will remain tachypneic and manifest paradoxical breathing for sometime after extubation due to the bony sternal defect.

\section{Conclusion}

Ectopia cordis is a rare congenital malformation. Its management requires prompt diagnosis, a quick referral to a tertiary health care facility, immediate resuscitation and monitoring in the ICU, and urgent surgical intervention. The prognosis is poor due to hypothermia during transfer, heart failure, hypoxemia, metabolic acidosis, desiccation of the viscera, infection, and sepsis. This is one such cardiac defect for which surgery is the only treatment option immediately after birth but with a high mortality. Implementation of ECMO may be of great help as a bridge to successful surgery for EC in future.

\section{Funding}

The authors did not receive any financial support for the research, authorship, and/or publication of this article.

\section{Conflict of Interest}

The authors declare that there are no potential conflicts of interest with respect to the research, authorship, and/or publication of this article.

\section{Acknowledgments}

We acknowledge the efforts and help provided by Dr. Anurag Goel and Dr. Abhisek for updating the videos and photos

\section{References}

1 Goncalo FIMD, Ana VRS, Catia FCPM, Ana PDF, Joaquim MDF. Ectopia cordis: caso clinic. Rev Bras Saúde Mater Infant 2014;14(3):287-290

2 Shad J, Budhwani K, Biswas R. Thoracic ectopia cordis. BMJ Case Rep 2012;2012:5241

3 Khoury MJ, Cordero JF, Rasmussen S. Ectopia cordis, midline defects and chromosome abnormalities: an epidemiologic perspective. Am J Med Genet 1988;30(3):811-817

4 Samir K, Ghez O, Metras D, Kreitmann B. Ectopia cordis, a successful single stage thoracoabdominal repair. Interact Cardiovasc Thorac Surg 2003;2(4):611-613

5 Alphonso N, Venugopal PS, Deshpande R, Anderson D. Complete thoracic ectopia cordis. Eur J Cardiothorac Surg 2003;23(3):426-428 
6 Morales JM, Patel SG, Duff JA, Villareal RL, Simpson JW. Ectopia cordis and other midline defects. Ann Thorac Surg 2000;70(1):111-114

7 Dobell ARC, Williams HB, Long RW. Staged repair of ectopia cordis. J Pediatr Surg 1982;17(4):353-358

8 Gabriel A, Donnelly J, Kuc A, et al. Ectopia cordis: a rare congenital anomaly. Clin Anat 2014;27(8):1193-1199

9 Grethel EJ, Hornberger LK, Farmer DL. Prenatal and postnatal management of a patient with pentalogy of Cantrell and left ventricular aneurysm. A case report and literature review. Fetal Diagn Ther 2007;22(4):269-273

10 Chandran S, Ari D. Pentalogy of Cantrell: an extremely rare congenital anomaly. J Clin Neonatol 2013;2(2):95-97

11 Harrison MR, Filly RA, Stanger P, de Lorimier AA. Prenatal diagnosis and management of omphalocele and ectopia cordis. J Pediatr Surg 1982;17(1):64-66

12 Cutler GD, Wilens G. Ectopia cordis: report of a case. Am J Dis Child 1925;30(1):76-81

13 Saxena AK. Pectus excavatum, pectus carinatum and other forms of thoracic deformities. J Indian Assoc Pediatr Surg 2005;10(3):147-157

14 Amato JJ, Zelen J, Talwalkar NG. Single-stage repair of thoracic ectopia cordis. Ann Thorac Surg 1995;59(2):518-520

15 Achiron R, Shimmel M, Farber B, Glaser J. Prenatal sonographic diagnosis and perinatal management of ectopia cordis. Ultrasound Obstet Gynecol 1991;1(6):431-434

16 Kumar B, Sharma C, Sinha DD, Sumanlata. Ectopia cordis associated with Cantrell's pentalogy. Ann Thorac Med 2008;3(4):152-153
17 Lopez JA, Lopez AG, Leon IH. Presentation and discussion of a patient with pentalogia of Cantrell. Cuban Rev Obstet Ginecol 2004;30:2

18 Skandalakis JE, Gray SW, Rickets R. The anterior body wall. Embryology for Surgeons 1994;5:552-559

19 Sakasai Y, Thang BQ, Kanemoto S, et al. Staged repair of pentalogy of Cantrell with ectopia cordis and ventricular septal defect. J Card Surg 2012;27(3):390-392

20 van Hoorn JHL, Moonen RM, Huysentruyt CJ, van Heurn LW, Offermans JP, Mulder AL. Pentalogy of Cantrell: two patients and a review to determine prognostic factors for optimal approach. Eur J Pediatr 2008;167(1):29-35

21 O'Gorman CS, Tortoriello TA, McMahon CJ. Outcome of children with Pentalogy of Cantrell following cardiac surgery. Pediatr Cardiol 2009;30(4):426-430

22 Hornberger LK, Colan SD, Lock JE, Wessel DL, Mayer JE Jr. Outcome of patients with ectopia cordis and significant intracardiac defects. Circulation 1996;94(9 Suppl):II32-II37

23 Leca F, Thibert M, Khoury W, Fermont L, Laborde F, Dumez Y. Extrathoracic heart (ectopia cordis). Report of two cases and review of the literature. Int J Cardiol 1989;22(2):221-228

24 Restrepo MS, Cerqua A, Turek JW. Pentalogy of Cantrell with ectopia cordis totalis, total anomalous pulmonary venous connection, and tetralogy of Fallot: a case report and review of the literature. Congenit Heart Dis 2014;9(4):E129-E134 